\title{
Single-Layer Circularly Polarized Wide Band Reflectarray Antenna with High Aperture Efficiency
}

\author{
Xinyu Da, ${ }^{1}$ Jialiang $W u\left(D,{ }^{2}\right.$ Jing Zhao, ${ }^{2}$ Lin Baoqin, ${ }^{2}$ and Kai $W u^{2}$ \\ ${ }^{1}$ Institute of Information Engineering, Yango University, Fuzhou, Fujian, China \\ ${ }^{2}$ Information and Navigation College, Airforce Engineering University, Xi'an, Shaanxi, China \\ Correspondence should be addressed to Jialiang Wu; wujia2538@126.com
}

Received 10 January 2018; Revised 5 May 2018; Accepted 3 June 2018; Published 3 July 2018

Academic Editor: Chien-Jen Wang

Copyright ( 2018 Xinyu Da et al. This is an open access article distributed under the Creative Commons Attribution License, which permits unrestricted use, distribution, and reproduction in any medium, provided the original work is properly cited.

A circularly polarized broadband low-cost reflectarray in Ku-band is presented using a novel single-layer subwavelength phaseshifting element. The proposed subwavelength element consists of the concentric split ring and the crossed bowtie. The linear reflected phase response curve with $360^{\circ}$ phase coverage is obtained. For experimental verification, an array of $25 \times 25$ reflectarray prototype has been designed and manufactured by employing the angular rotation technique. The measurements are in good agreement with the simulations. The measured gain at the center frequency of $12.5 \mathrm{GHz}$ is $26.6 \mathrm{dBi}$, corresponding to the aperture efficiency of $52.5 \%$, and the $1 \mathrm{~dB}$ gain bandwidth is $26.4 \%$.

\section{Introduction}

High-gain antennas with narrow half-power beamwidth (HPBW) are desired for long-distance communications, such as satellite commutations, deep space exploration, radar detection, radio astronomy, and military application. In the past few decades, parabolic reflectors and phase arrays have been normally designed for these requirements. Parabolic reflectors possess some advantages such as low cost, simple geometric construction, and well developed. Nevertheless, the parabolic reflectors have nonplanar curved surface that renders it bulky for particular applications. In addition, the beam scanning is mainly based on mechanical rotation, which makes it not flexible enough to meet the increasing demands. On the other hand, traditional phase arrays have been widespread used in many applications due to its planar geometric structure, fast beam scanning and beam forming, low profile, and so on. However, these traditional phase arrays require complex feeding networks, leading to the mass antenna losses caused by the long path of transmission lines for RF signals. Besides, the considerable losses caused by network bends and discontinuities affect the radiation performances of large phase array antennas operating at high frequencies [1]. Recently, the planar surface reflectarray
(RA) antennas, which combine some favorable features of both parabolic reflectors and phase arrays, have attracted significant attention. RA has been regarded as a new generation of high-gain antenna because of its merits such as high efficiency, simplified structure, easier to design and manufacture, and lightweight [2-4].

For all that, one of the main limitations of RA antenna technology is its limited bandwidth which is primarily bounded by the narrowband of elements and differential spatial phase errors [5]. Hence, special attentions have been paid to design various broadband and multiband RA antennas, such as using stacked patches with variable-length phase delay lines, aperture-coupled patches, and multilayer patch elements [6-9]. However, more efforts have been devoted to propose single-layer elements, especially the single-layer multiresonant elements. Compared with the multilayer patch elements, single-layer patch elements have the simpler geometric structures and more convenient fabrication process. Moreover, by combining two or more simple structures appropriately, more resonances of the single-layer multiresonant elements can be introduced to broaden the bandwidth [10-15]. Hence, some novel patch elements have been presented in the literatures, such as with double cross loops [10], combination of cross and rectangle loop [11], the phoenix 
cell [12], triple rectangular loops [13], combination of cross loop and two circular rings [14], triple square rings, and combined square ring and I-shape strip [15] to design RA antennas.

Circularly polarized (CP) antennas are preferred in many applications because of the feasibility against environment interference, and various $\mathrm{CP}$ reflectarray antennas have been implemented to meet the increasing demands. The CP reflectarray antennas are principally designed by using variable patch size and angular rotation technique. In [16-21], reflectarray antennas with linear polarization or circular polarization feeders have been designed to attain the $\mathrm{CP}$ focused beam. By adjusting the dimension of each patch, the incident wave can be decomposed into two equal orthogonal components with $90^{\circ}$ phase difference, and the CP focused beam can be obtained. However, the main drawback of the reflectarray antennas using variable size technique is that the bandwidth is still relatively narrow. In [22-28], the angular rotation technique has been applied to implement RA antenna designs for overcoming such drawback. It is used to compensate the phase shift to attain $\mathrm{CP}$ focused beam. One prerequisite condition of angular rotation technique is that the primary source antenna must be a CP feeder.

In this work, a novel single-layer patch element has been proposed to design the wideband high-efficiency $\mathrm{CP}$ reflectarray antenna. Ansoft HFSS has been employed to optimize the parameters and investigate the radiation performances of element. The full $360^{\circ}$ phase coverage has been achieved by using the angular rotation technique, and the reflection loss has been also considered. A reflectarray prototype of the proposed structure has been designed, manufactured, and measured. The radiation performances of proposed reflectarray antenna are better than the previous reflectarray works in terms of gain bandwidth, axial ratio bandwidth, and efficiency.

\section{Element Analysis and Design}

The geometric structure of the proposed novel phasing element is depicted in Figure 1. It is printed on the $1.6 \mathrm{~mm}$ grounded substrate with the relative dielectric constant $\varepsilon_{\mathrm{r}}=$ 2.65 and the $\tan \delta=0.001$. For the sake of achieving the smooth phase variations to obtain better radiation performance of RA antenna, the size of unit cell is recommended to be minimized as possible. However, the narrow gap between adjacent elements will introduce phase error, which is unfavorable to design antenna. Hence, the patch element is constructed on the subwavelength unit cell with the grid spacing of $p=8 \mathrm{~m}$ $\mathrm{m}(1 / 3 \lambda$ when operating at $12.5 \mathrm{GHz})$, instead of the conventional $1 / 2 \lambda$ size unit cell, where $\lambda$ is the wavelength in free space at central frequency. The element consists of two segments. The outer part is the concentric split ring, which is characterized by its parameter outer ring radius $r_{\mathrm{o}}$, line width $w_{\mathrm{o}}$, and gap width $g_{0}$. The inner part is the crossed bowtie, which is characterized by its parameter bowtie length $l_{1}$ and $l_{2}$, span angle $\alpha_{1}$ and $\alpha_{2}$, and rotation angle $\beta$. The optimized size parameters are depicted in Figure 1.

Figure 2 shows the operating principle of $\mathrm{CP}-\mathrm{CP}$ transmission with angular rotation technique. Take an incident normal left-hand circularly polarized wave $\vec{E}^{1}$ as to be

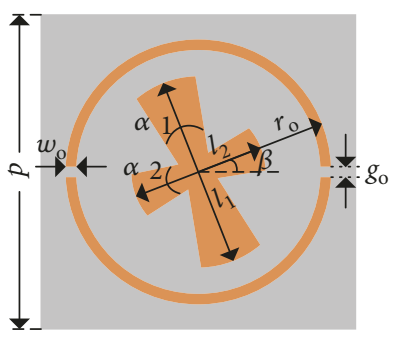

FIgURE 1: Configurations of the proposed single-layer subwavelength combined split ring and crossed bowtie element. The optimized size parameters are $r_{\mathrm{o}}=3.4 \mathrm{~mm}, w_{\mathrm{o}}=g_{\mathrm{o}}=0.25 \mathrm{~m}$ $\mathrm{m}, l_{1}=5 \mathrm{~mm}, l_{2}=3.5 \mathrm{~mm}, \alpha_{1}=\alpha_{2}=40^{\circ}$, and $\beta=21^{\circ}$.

considered, which travels towards the $z$ direction onto the reflectarray element. The electric field vector of the incident wave $\vec{E}^{1}$ and reflected wave $\vec{E}^{\mathrm{r}}$ can be expressed as

$$
\begin{aligned}
& \vec{E}^{\mathrm{i}}=\left(\widehat{a}_{x}+j \widehat{a}_{y}\right) A e^{-j k z} e^{-j \omega t}, \\
& \vec{E}^{\mathrm{r}}=\left(\widehat{a}_{x} e^{j \phi_{x}}+j \widehat{a}_{y} e^{j \phi_{y}}\right) A e^{j k z} e^{-j \omega t} .
\end{aligned}
$$

When the element is counterclockwise rotated with $\psi$ degree, the incident wave $\vec{E}^{1}$ and the reflected wave $\vec{E}^{\mathrm{r}}$ can be rewritten as

$$
\begin{aligned}
\vec{E}^{\mathrm{i}}= & {\left[\left(\widehat{a}_{x^{\prime}} \cos \psi-\widehat{a}_{y^{\prime}} \sin \psi\right)\right.} \\
& \left.+j\left(\widehat{a}_{x^{\prime}} \sin \psi+\widehat{a}_{y^{\prime}} \cos \psi\right)\right] A e^{-j k z} e^{-j \omega t} \\
= & {\left[\widehat{a}_{x^{\prime}}\left(\frac{e^{j \psi}+e^{-j \psi}}{2}+j \frac{e^{j \psi}-e^{-j \psi}}{2 j}\right)\right.} \\
& \left.-\widehat{a}_{y^{\prime}}\left(\frac{e^{j \psi}-e^{-j \psi}}{2 j}-j \frac{e^{j \psi}+e^{-j \psi}}{2}\right)\right] A e^{-j k z} e^{-j \omega t} \\
= & \left(\widehat{a}_{x^{\prime}}+j \widehat{a}_{y^{\prime}}\right) A e^{-j k z} e^{-j \omega t} e^{j \psi}, \\
\vec{E}^{r}= & \left(\widehat{a}_{x^{\prime}} e^{j \phi_{x^{\prime}}}+j \widehat{a}_{y^{\prime}} e^{j \phi_{y^{\prime}}}\right) A e^{j k z} e^{-j \omega t} e^{j \psi} \\
= & {\left[\left(\widehat{a}_{x} \cos \psi+\widehat{a}_{y} \sin \psi\right) e^{j \phi_{x}}\right.} \\
& \left.+j\left(-\widehat{a}_{x} \sin \psi+\widehat{a}_{y} \cos \psi\right) e^{j \phi_{y}}\right] A e^{j k z} e^{-j \omega t} e^{j \psi} \\
= & \frac{A}{2}\left[\left(\widehat{a}_{x}+j \widehat{a}_{y}\right)\left(e^{j \phi_{x}}+e^{j \phi_{y}}\right)\right. \\
& \left.+\left(\widehat{a}_{x}-j \widehat{a}_{y}\right)\left(e^{j \phi_{x}}-e^{j \phi_{y}}\right) e^{j 2 \psi}\right] e^{j k z} e^{-j \omega t} .
\end{aligned}
$$

When the difference between phase shift of reflected wave $\phi_{x}$ and $\phi_{y}$ is $\pm \pi$, the reflected wave can be expressed as $\vec{E}^{\mathrm{r}}=A\left(\widehat{a}_{x}-j \widehat{a}_{y}\right) e^{j \phi_{x}} e^{j k z} e^{-j \omega t} e^{j 2 \psi}$, and the left-hand circularly polarized reflected wave can be obtained.

The commercial software Ansoft HFSS has been applied to evaluate the radiation performance of element. Periodic boundaries along $x$ and $y$ directions and Floquet mode excitations are both introduced to accomplish the infinitearray approach for element analysis. For obtaining the reflection phasing characteristic of each corresponding element, left-hand circularly polarized (LHCP) plane wave which is normal incidence on an infinite periodic array has 

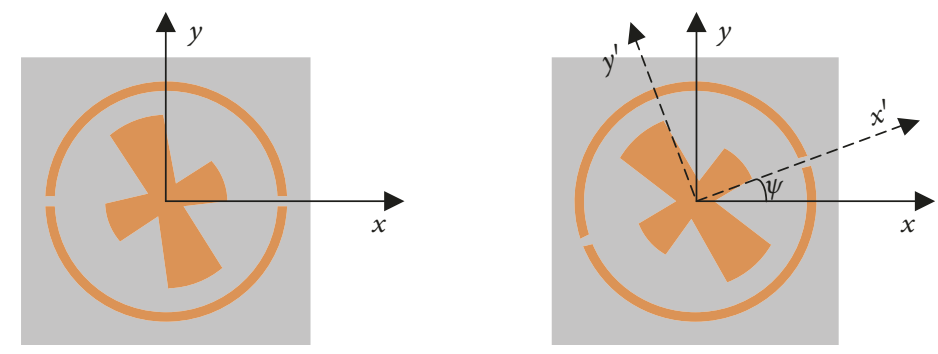

FIGURE 2: Demonstration of the CP-CP transformation with angular rotation technique.

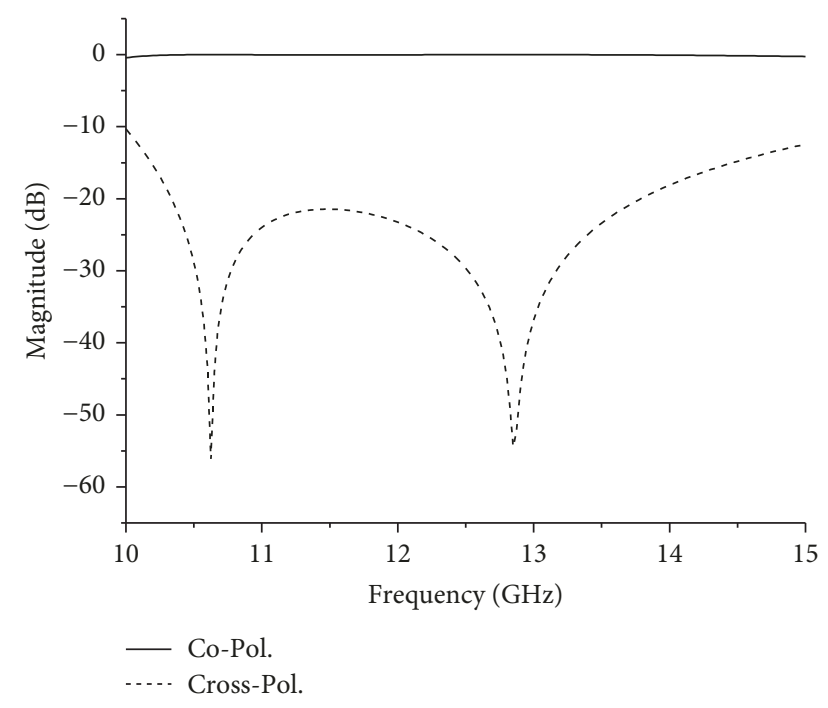

FIGURE 3: Copolarization and cross-polarization component magnitude of the proposed element versus frequency under normal incidence.

been considered. Two linearly polarized plane waves with $90^{\circ}$ phase difference are introduced to obtain the LHCP wave. Before element rotation, the magnitudes of both LHCP and right-hand circularly polarized (RHCP) component around the center frequency are first considered. It can be observed clearly from Figure 3 that the proposed element possesses the potential to operate in a broad band with low losses in the whole operating frequency bandwidth. In order to evaluate the sensitivity of the proposed element under different incident angles, the copolarization (LHCP) and cross-polarization (RHCP) component magnitudes versus incident angle have been investigated around the center frequency, and the magnitude curves are depicted in Figures 4 and 5, respectively. As shown in Figures 4 and 5, the magnitude of cross-polarization component is still in a small value, while the maximum amplitude loss occurs about $0.4 \mathrm{~dB}$ with multiple incident angles in the whole operating frequency range, further confirming the ability of the proposed element to provide the wider bandwidth for $\mathrm{CP}$ reflectarray antenna design.

Before designing the $\mathrm{CP}$ reflectarray surface, the reflected phases with varying element rotation angle under multiple incident angles have been studied. Figure 6 shows that the reflected phase curves satisfy the $360^{\circ}$ with relative linear variation. Besides, the accurate results can be carried out when

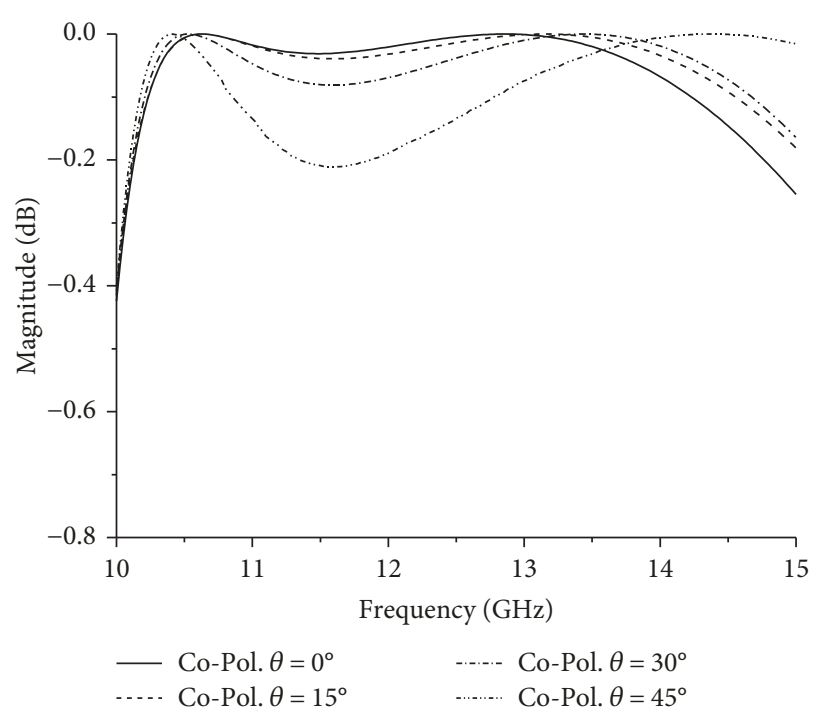

FIGURE 4: Copolarization component magnitudes of the proposed element versus frequency under multiple incidence angles.

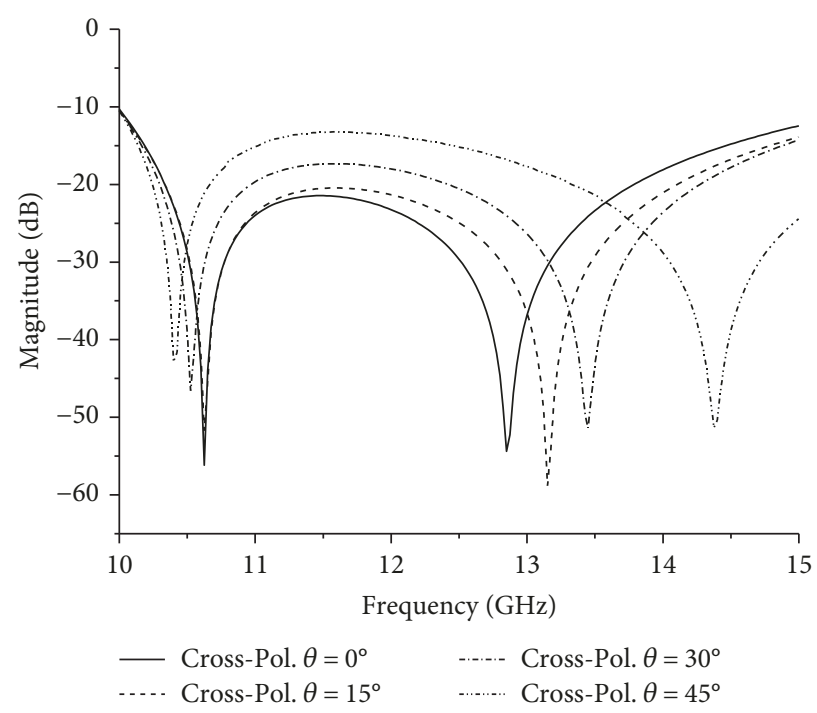

Figure 5: Cross-polarization component magnitudes of the proposed element versus frequency under multiple incidence angles.

angle of incident EM wave is taken into account during $\mathrm{CP}$ reflectarray surface design. The rotation angle of each individual element can be obtained for compensating the phase 


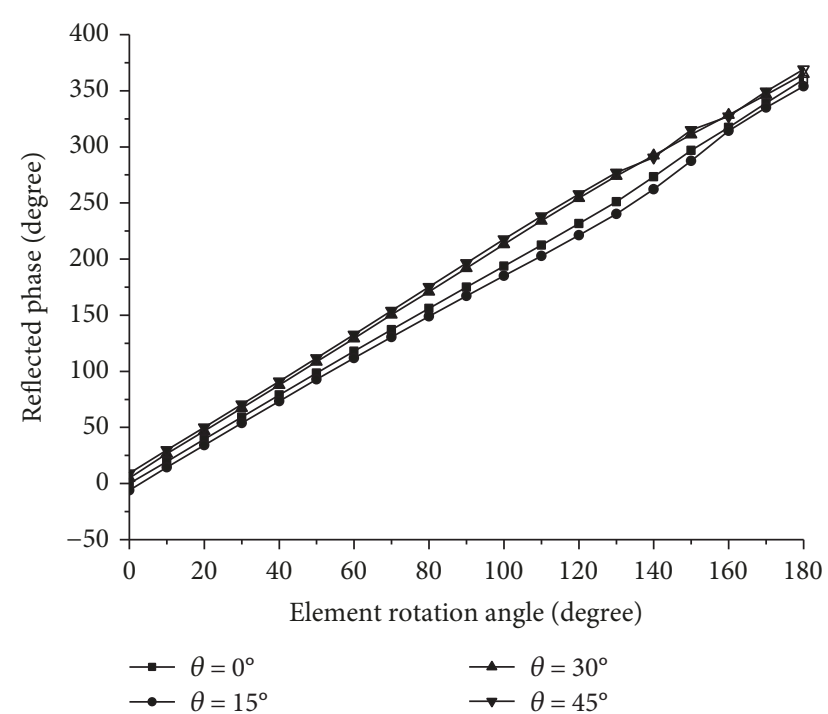

Figure 6: Reflected phase responses versus element rotation angle under multiple incident angles at $12.5 \mathrm{GHz}$.

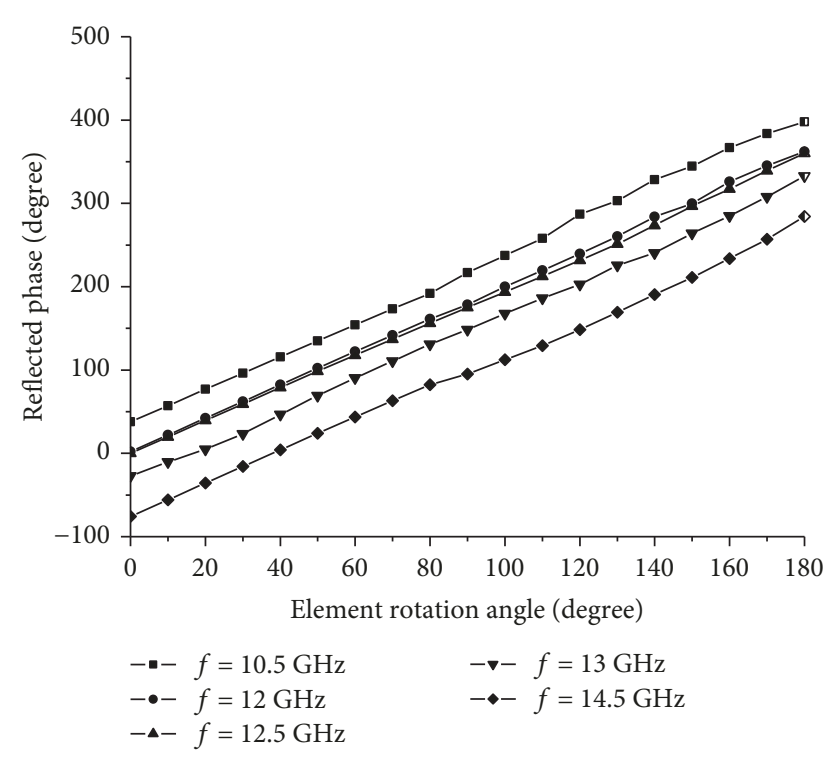

FIGURE 7: Reflected phase responses versus element rotation angle under normal incidence at different frequencies.

delay under multiple incident angles. In addition, in order to forecast the possible wide bandwidth of the $\mathrm{CP}$ reflectarray surface, the reflected phase variation of the proposed element versus element rotation angle at different frequencies is depicted in Figure 7. The phase curves clearly indicate that in the operating frequency range, the phase variation of each element location at different frequency will be within $\pm 90^{\circ}$, indicating that the wide bandwidth of $\mathrm{CP}$ reflectarray surface with the proposed element can be achieved. Moreover, the reflection coefficient magnitudes under normal and oblique incidence of the proposed element at the center frequency have been plotted in Figure 8. The curves demonstrate that the reflected wave magnitude is close to $0 \mathrm{~dB}$, which means that the incident voltage is almost equal to the reflected

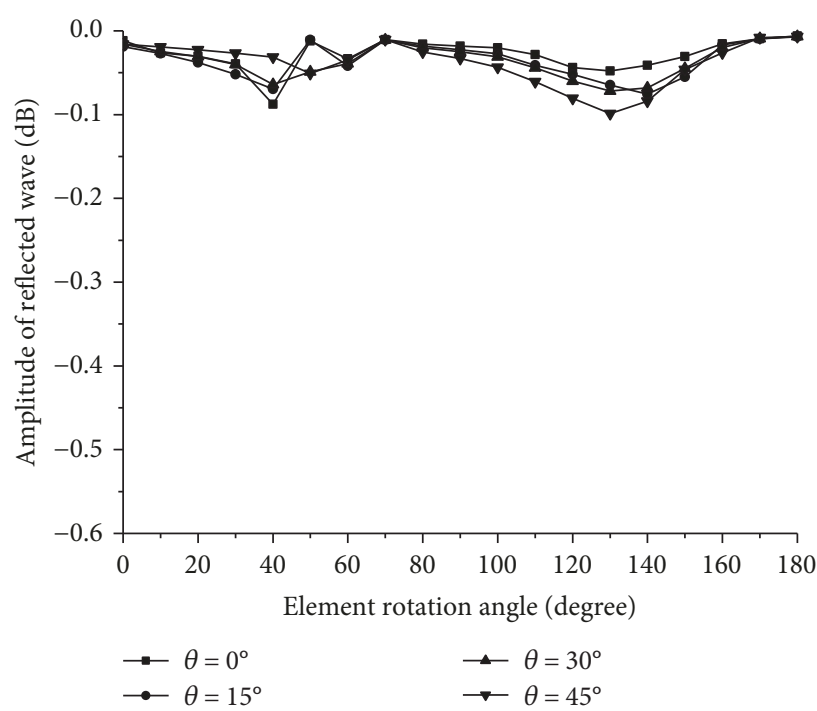

FIGURE 8: Reflection coefficient magnitude of the proposed element under normal incidence at $12.5 \mathrm{GHz}$.

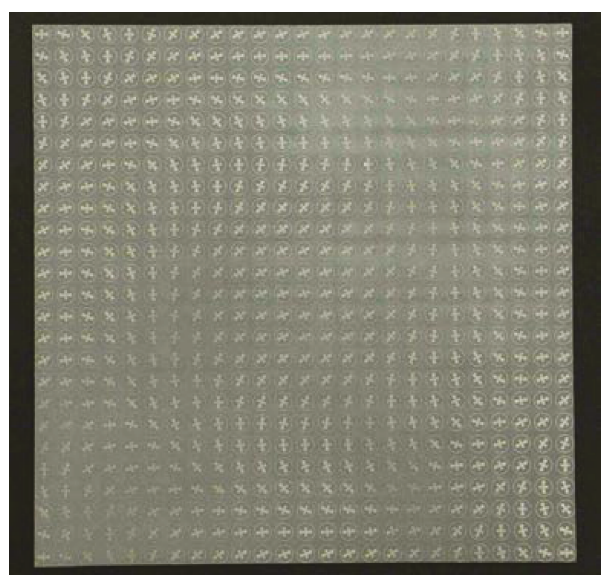

Figure 9: Photograph of reflectarray prototype.

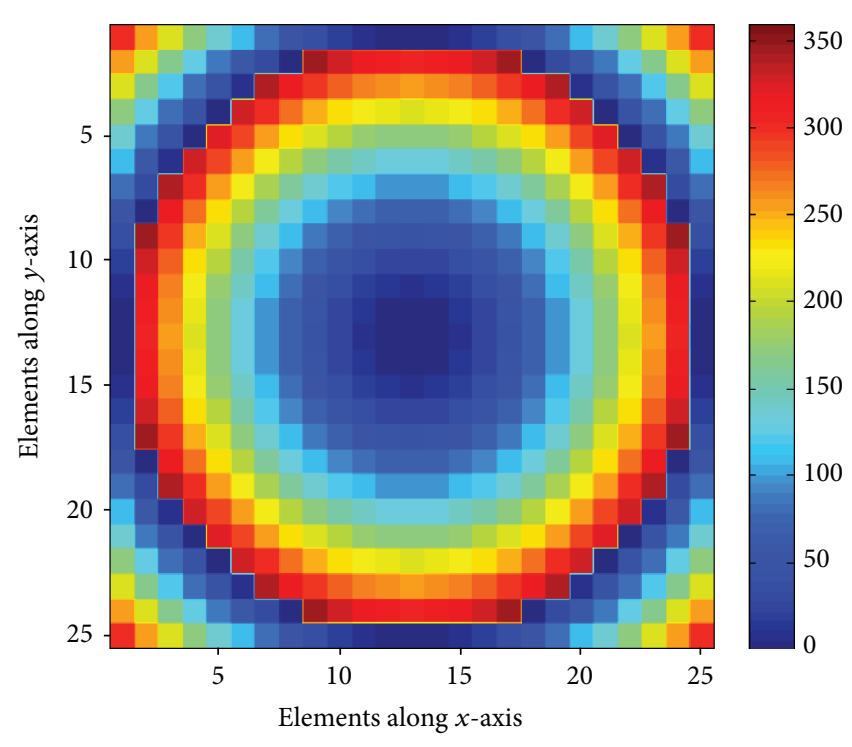

FIgURE 10: Required phase distribution to compensate spatial delay of each element at $12.5 \mathrm{GHz}$. 


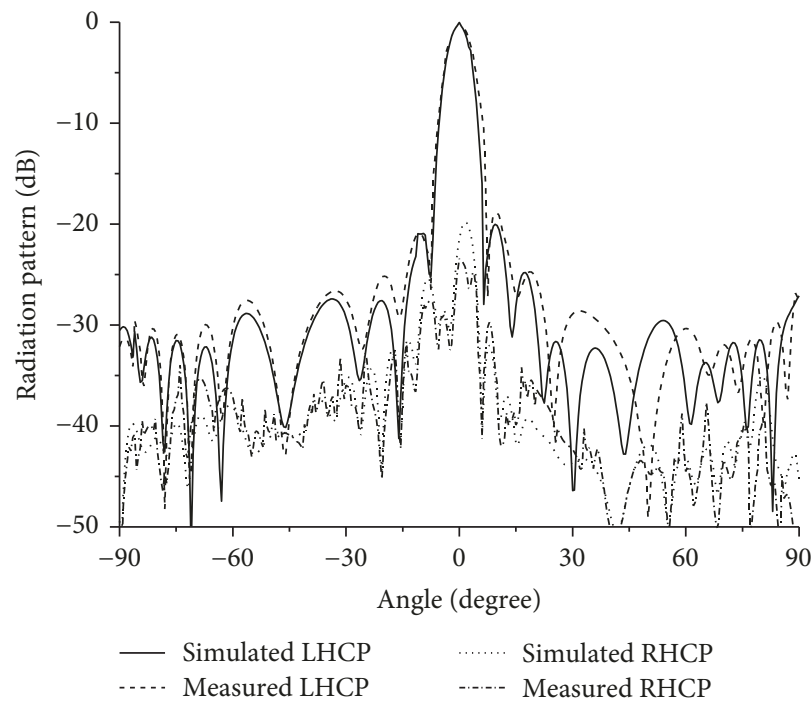

(a)

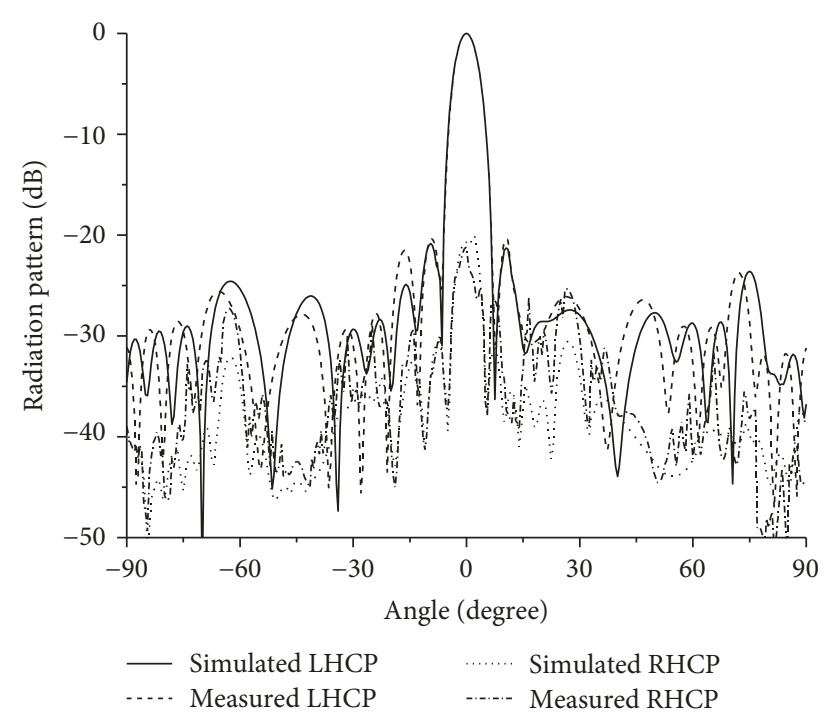

(b)

FIGURE 11: Measured and simulated far-field copolarization and cross-polarization radiation patterns in $x-z$ and $y-z$ planes at $12.5 \mathrm{GHz}$ : (a) $x$ - $z$ plane and (b) $y$ - $z$ plane.

one, and the grating lobes or surface waves generated from ground plane will be minimized. All the analyses mentioned above can conclude that the proposed element is suitable for $\mathrm{CP}$ reflectarray antenna design.

\section{Realization of Reflectarray and Performance}

To verify the radiation performance of the proposed element mentioned above, an array of $25 \times 25 \mathrm{RA}$ prototype has been designed for analysis, and it has been fabricated by printed board technique, as shown in Figure 9. The center-fed RA prototype has been designed at $12.5 \mathrm{GHz}$. The dimension of the square RA aperture is $20 \times 20 \mathrm{~cm}^{2}$, and the element spacing is considered at $1 / 3 \lambda$. The fabricated RA prototype has been measured by using the compact-range anechoic chamber. A circular polarized conical horn antenna, which is located $20 \mathrm{~cm}$ above the center of RA aperture, has been chosen as the feed, giving the $F / D$ ratio equal to 1 .

Considering that the feed horn antenna has been located far enough to the RA prototype, the normal incident waves generated from feed horn antenna can be taken as the plane waves. Element rotation technique is used to compensate the required phase shift of each individual element. In view of that the RA prototype in the $x-y$ plane is located with the phase center of $\left(x_{f}, y_{f}, z_{f}\right)$, a numerical MATLAB code has been implemented to determine the required phase distribution $\psi\left(x_{i}, y_{i}\right)$ at each element to collimate the required beam towards $\left(\theta_{0}, \varphi_{0}\right)$ direction. The required phase distribution of each element can be calculated by using (1) and (2).

$$
\begin{aligned}
\psi\left(x_{i}, y_{i}\right) & =k_{0}\left(d_{i}-\sin \theta_{\mathrm{o}}\left(x_{i} \cos \varphi_{\mathrm{o}}+y_{i} \sin \varphi_{\mathrm{o}}\right)\right)+2 \pi N, \\
d_{i} & =\sqrt{\left(x_{i}-x_{f}\right)^{2}+\left(y_{i}-y_{f}\right)^{2}+\left(z_{f}\right)^{2}}
\end{aligned}
$$

where $k_{0}$ is the propagation constant in the free space, $d_{i}$ represents the spatial distance between the center of feed antenna and the $i$ th element of RA aperture, and $\left(x_{i}, y_{i}\right)$ stands for the coordinates of $i$ th element center. Hence, the required phase distribution to compensate spatial delay of each element can be calculated, as shown in Figure 10. The phase response curves under multiple incidences mentioned above can be used to determine the rotation angles of the radiating elements, and the optimization design of RA aperture can be accomplished.

The simulated and measured far-field copolarization and cross-polarization radiation patterns of the RA in both $x-z$ and $y-z$ planes at $12.5 \mathrm{GHz}$ are plotted in Figure 11 . By comparing the simulated and measured radiation patterns, it can be observed that the overall radiation characteristic agrees well. The pencil-shaped beams are well defined in the predefined directions, while the sidelobes match well. Hence, it can be concluded that the measured radiation patterns of the RA antenna are in good agreement with the simulated ones. The HPBW in $x-z$ and $y-z$ planes are $7^{\circ}$ and $6^{\circ}$, respectively. As shown in Figure 11(a), the measured peak side lobe level (SLL) is $19 \mathrm{~dB}$ below the peak gain in $x-z$ plane while the peak cross-polarization level is near $-24 \mathrm{~dB}$. As shown in Figure 11(b), the measured SLL is at $-20 \mathrm{~dB}$ in $y-z$ plane while the crosspolarization levels are all below $-21 \mathrm{~dB}$ in boresight.

The simulated and measured gain variation in the boresight direction versus frequency bands of interest is presented in Figure 12. The maximum measured gain is about $26.7 \mathrm{dBi}$ appearing at $12.6 \mathrm{GHz}$. The simulated gain at $12.5 \mathrm{GHz}$ is about $27.6 \mathrm{dBi}$ while the measured one is about $26.6 \mathrm{dBi}$. The difference between the simulation and measurement is $1 \mathrm{dBi}$ which is acceptable in practical application. The measured bandwidth of $1 \mathrm{~dB}$ gain drop is from $11.2 \mathrm{GHz}$ to $14.5 \mathrm{GHz}$, corresponding to $26.4 \%$ bandwidth with respect to $12.5 \mathrm{GHz}$. The simulated and measured axial ratio (AR) 


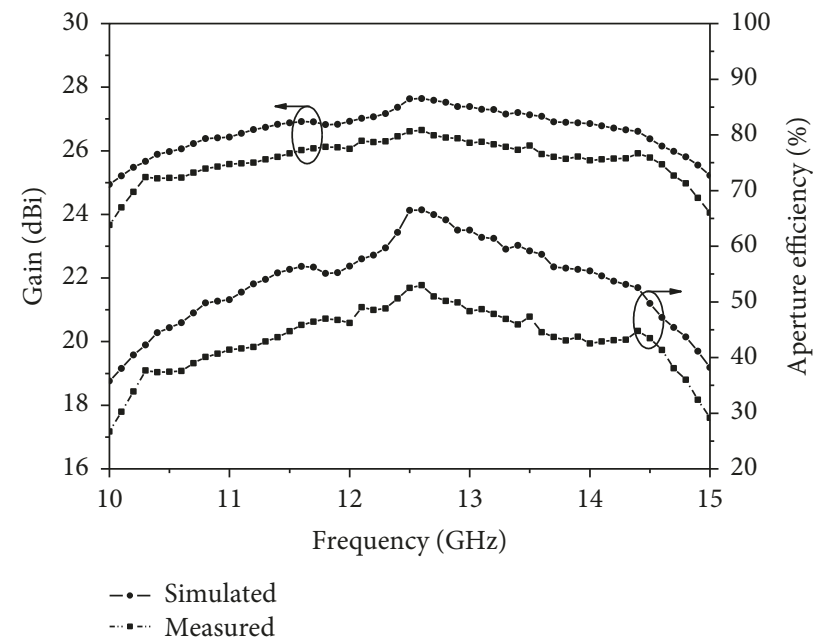

FIGURE 12: Measured and simulated gain and aperture efficiency at boresight direction versus frequency.

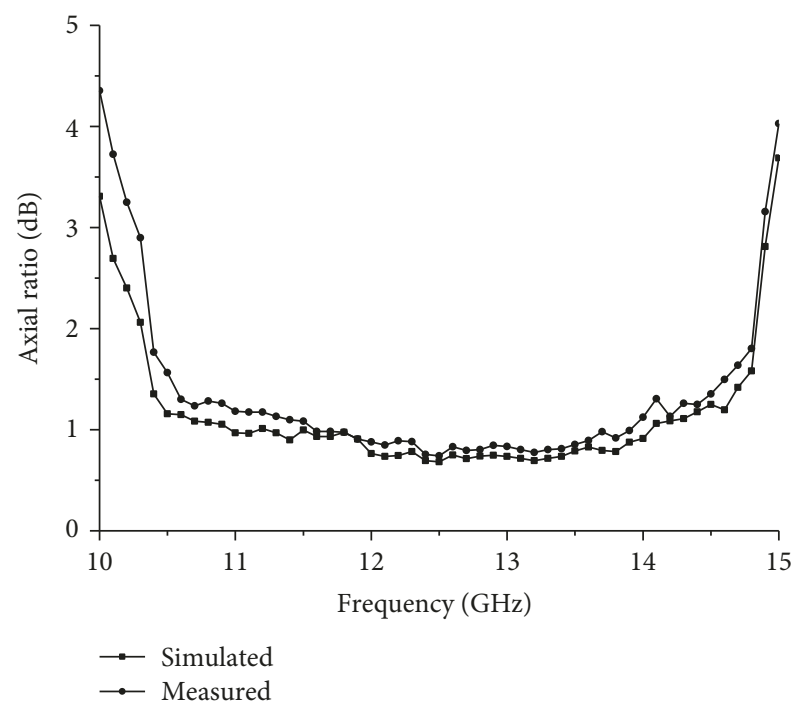

Figure 13: Measured and simulated axial ratio variation versus frequency bands of interest.

variation versus frequency bands of interest is shown in Figure 13. The simulation and measurement agree well. The measured $3 \mathrm{~dB}$ AR bandwidth is $36 \%$ from 10.3 to 14.8 GHz, while the $1 \mathrm{~dB}$ AR bandwidth is $18.4 \%$ from 11.6 to $13.9 \mathrm{GHz}$.

The simulated and measured aperture efficiencies have been calculated based on the gains with the following formula:

$$
\eta=\frac{\text { gain } \times \lambda^{2}}{4 \pi \times \text { area }},
$$

where area represents the physical area of the RA aperture. The measured aperture efficiency is about $52.5 \%$ at $12.5 \mathrm{GHz}$, and the maximum is about $53 \%$ at $12.6 \mathrm{GHz}$, indicating the excellent radiation performances of the proposed RA antenna. It should be pointed out that the random phase errors, losses in elements and substrates, blockages of feeding
TABLE 1: Comparison of previous RA performance with the proposed.

\begin{tabular}{lcccc}
\hline Work & $\begin{array}{c}\text { Central } \\
\text { freq. }\end{array}$ & $\begin{array}{c}\text { Gain BW } \\
1 \mathrm{~dB}\end{array}$ & $\begin{array}{c}\text { AR BW } \\
3 \mathrm{~dB}\end{array}$ & $\begin{array}{c}\text { AR BW } \\
1 \mathrm{~dB}\end{array}$ \\
\hline$[24]$ & 10 & $17 \%$ & $30 \%$ & $18 \%$ \\
{$[25]$} & 10 & $19.1 \%$ & $40 \%$ & - \\
{$[26]$} & 11.8 & $10 \%$ & $14.6 \%$ & $10 \%$ \\
{$[27]$} & 13.5 & $11.1 \%$ & $20.3 \%$ & - \\
{$[28]$} & 15 & $19.3 \%$ & $15 \%$ & - \\
This & 12.5 & $26.4 \%$ & $36 \%$ & $18.4 \%$ \\
work & & & &
\end{tabular}

horn, and measurement errors could also reduce the aperture efficiencies of the two proposed RA antennas. Note that compared with the previous reflectarray works [24-28], the proposed reflectarray antenna has better radiation performances in terms of $3 \mathrm{~dB}$ AR bandwidth, $1 \mathrm{~dB}$ gain bandwidth, and aperture efficiency, as shown in Table 1.

\section{Conclusion}

This work has presented a Ku-band circularly polarized reflectarray model with the novel combined split ring and crossed bowtie element. The reflected phase range with full $360^{\circ}$ phase coverage has been achieved by rotating the proposed element. An array of $25 \times 25$ reflectarray prototype has been designed, manufactured, and measured at the center frequency of $12.5 \mathrm{GHz}$. The measured SLL is at $-20 \mathrm{~dB}$, and the cross-polarization levels are all below $-21 \mathrm{~dB}$. The measured gain at $12.5 \mathrm{GHz}$ is $26.6 \mathrm{dBi}$, corresponding to the aperture efficiency of $52.5 \%$. The measured $1 \mathrm{~dB}$ gain bandwidth is $26.4 \%$, while the $3 \mathrm{~dB}$ AR bandwidth is $36 \%$.

\section{Data Availability}

The data that support the findings of this study are available from the corresponding author on request.

\section{Conflicts of Interest}

The authors declare that they have no conflicts of interest.

\section{Acknowledgments}

The authors are grateful to the supports from the National Natural Science Foundation of China under Grant nos. 61471387,61271250 , and 61571460.

\section{References}

[1] D. M. Pozar, S. D. Targonski, and H. D. Syrigos, "Design of millimeter wave microstrip reflectarrays," IEEE Transactions on Antennas and Propagation, vol. 45, no. 2, pp. 287-296, 1997.

[2] S. Xua and F. Yanga, Reflectarray Antennas, Tsinghua University, Beijing, China, 2015. 
[3] J. Huang and J. A. Encinar, Reflectarray Antennas, by Institute of Electrical and Electronics Engineers, Wiley, Hoboken, NJ, 2008.

[4] J. Shaker, M. R. Chaharmir, and J. Ethier, Reflectarray Antennas: Analysis, Design, Fabrication and Measurement, Artech House, Norwood, MA, USA, 2014.

[5] D. M. Pozar, "Bandwidth of reflectarrays," Electronics Letters, vol. 39, no. 21, pp. 1490-1491, 2003.

[6] E. Carrasco, J. É. A. Encinar, and M. Barba, "Bandwidth improvement in large reflectarrays by using true-time delay," IEEE Transactions on Antennas and Propagation, vol. 56, no. 8, pp. 2496-2503, 2008.

[7] J. A. Encinar and J. A. Zornoza, "Broadband design of threelayer printed reflectarrays," IEEE Transactions on Antennas and Propagation, vol. 51, no. 7, pp. 1662-1664, 2003.

[8] P. Nayeri, F. Yang, and A. Z. Elsherbeni, "Broadband reflectarray antennas using double-layer subwavelength patch elements," IEEE Antennas and Wireless Propagation Letters, vol. 9, pp. 1139-1142, 2010.

[9] L.-S. Ren, Y.-C. Jiao, F. Li, J.-J. Zhao, and G. Zhao, "A duallayer T-shaped element for broadband circularly polarized reflectarray with linearly polarized feed," IEEE Antennas and Wireless Propagation Letters, vol. 10, pp. 407-410, 2011.

[10] M. R. Chaharmir, J. Shaker, M. Cuhaci, and A. Ittipiboon, "Broadband reflectarray antenna with double cross loops," Electronics Letters, vol. 42, no. 2, pp. 65-66, 2006.

[11] M. R. Chaharmir and J. Shaker, "Broadband reflectarray with combination of cross and rectangle loop elements," Electronics Letters, vol. 44, no. 11, pp. 658-659, 2008.

[12] L. Moustafa, R. Gillard, F. Peris, R. Loison, H. Legay, and E. Girard, "The phoenix cell: a new reflectarray cell with large bandwidth and rebirth capabilities," IEEE Antennas and Wireless Propagation Letters, vol. 10, pp. 71-74, 2011.

[13] M. Mohammadirad, N. Komjani, M. R. Chaharmir, J. Shaker, and A. R. Sebak, "Impact of feed position on the operating band of broadband reflectarray antenna," IEEE Antennas and Wireless Propagation Letters, vol. 11, pp. 1104-1107, 2012.

[14] M. N. Jazi, M. R. Chaharmir, J. Shaker, and A. R. Sebak, "Reflectarray antennas using single layer polarization independent multi-resonant unit cells," in 2014 IEEE Antennas and Propagation Society International Symposium (APSURSI), pp. 805-806, Memphis, TN, USA, July 2014.

[15] M. Niroo-Jazi, M. R. Chaharmir, J. Shaker, and A. R. S. Author, "On radiation performances of reflectarray antennas constructed with subwavelength unit cells," IEEE Antennas and Wireless Propagation Letters, vol. 14, pp. 1258-1262, 2015.

[16] F. Ahmadi, A. Namiranian, and B. Virdee, "Design and implementation of a single layer circularly polarized reflectarray antenna with linearly polarized feed," Electromagnetics, vol. 35, no. 2, pp. 93-100, 2015.

[17] Y.-Y. Chen, Y. Ge, and T. S. Bird, "An offset reflectarray antenna for multipolarization applications," IEEE Antennas and Wireless Propagation Letters, vol. 15, pp. 1353-1356, 2016.

[18] C. Tian, Y. C. Jiao, and G. Zhao, "Circularly polarized transmitarray antenna using low-profile dual-linearly polarized elements," IEEE Antennas and Wireless Propagation Letters, vol. 16, pp. 465-468, 2017.

[19] L. Di Palma, A. Clemente, L. Dussopt, R. Sauleau, P. Potier, and P. Pouliguen, "Circularly polarized transmitarray with sequential rotation in ka-band," IEEE Transactions on Antennas and Propagation, vol. 63, no. 11, pp. 5118-5124, 2015.
[20] M.-Y. Zhao, G. Q. Zhang, X. Lei, J. M. Wu, and J. Y. Shang, "Design of new single-layer multiple-resonance broadband circularly polarized reflectarrays," IEEE Antennas and Wireless Propagation Letters, vol. 12, pp. 356-359, 2013.

[21] L. Guo, P.-K. Tan, and T.-H. Chio, "On the use of single-layered subwavelength rectangular patch elements for broadband folded reflectarrays," IEEE Antennas and Wireless Propagation Letters, vol. 16, pp. 424-427, 2017.

[22] M. M. Tahseen and A. A. Kishk, "Ka-band circularly polarized high efficiency wide band reflectarray using cross bow-tie elements," Progress In Electromagnetics Research, vol. 153, pp. 1-10, 2015.

[23] A.-H. Mahmoud and A. A. Kishk, "Ka-band low profile circularly polarized reflectarray," Progress In Electromagnetics Research C, vol. 63, pp. 43-51, 2016.

[24] G. Zhao, Y. C. Jiao, F. Zhang, and F. S. Zhang, "A subwavelength element for broadband circularly polarized reflectarrays," IEEE Antennas and Wireless Propagation Letters, vol. 9, pp. 330-333, 2010.

[25] X.-J. Zhong, L. Chen, Y. Shi, and X. W. Shi, "Single-layer broadband circularly polarized reflectarray with subwavelength double-ring elements," Electromagnetics, vol. 35, no. 4, pp. 217-226, 2015.

[26] R. S. Malfajani and Z. Atlasbaf, "Design and implementation of a broadband single layer circularly polarized reflectarray antenna," IEEE Antennas and Wireless Propagation Letters, vol. 11, pp. 973-976, 2012.

[27] X.-J. Zhong, L. Chen, Y. Shi, and X. W. Shi, "A dual-band single-layer circularly polarized reflectarray composed by elements having variable rotation angles," International Journal of RF and Microwave Computer-Aided Engineering, vol. 25, no. 1, pp. 39-47, 2015.

[28] J. Guo, F. Wu, and J. Wang, "Design of broadband circularly polarized reflectarrays with novel ring-stub elements," in 2015 Asia-Pacific Microwave Conference (APMC), vol. 3, pp. 1-3, Nanjing, China, December 2015. 


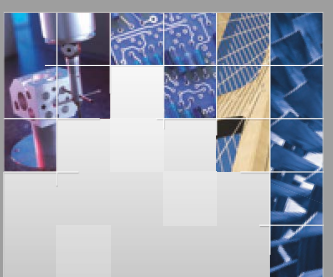

\section{Enfincering}
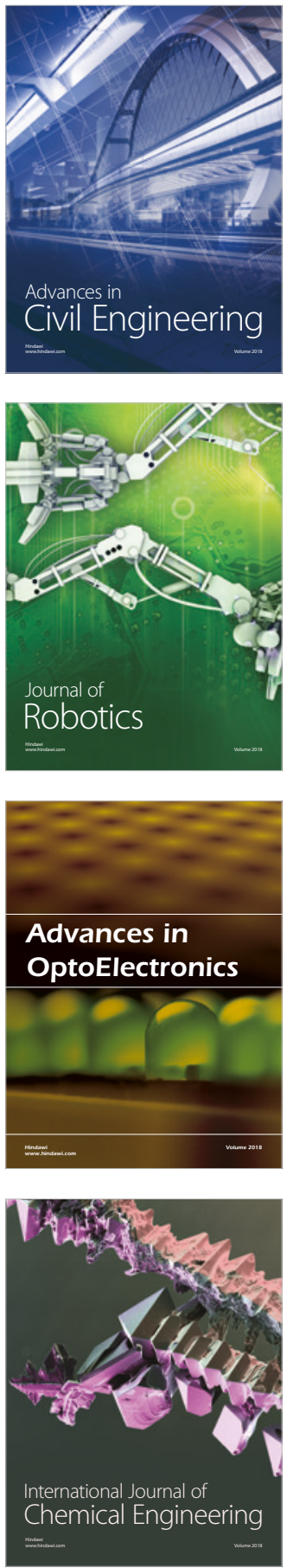

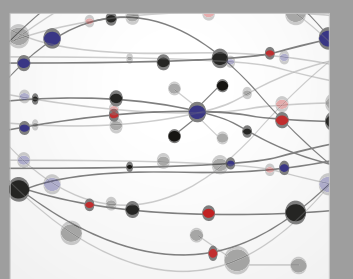

\section{Rotating \\ Machinery}

The Scientific World Journal

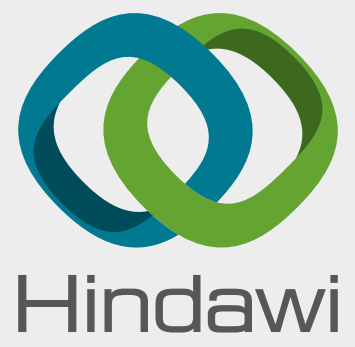

Submit your manuscripts at

www.hindawi.com
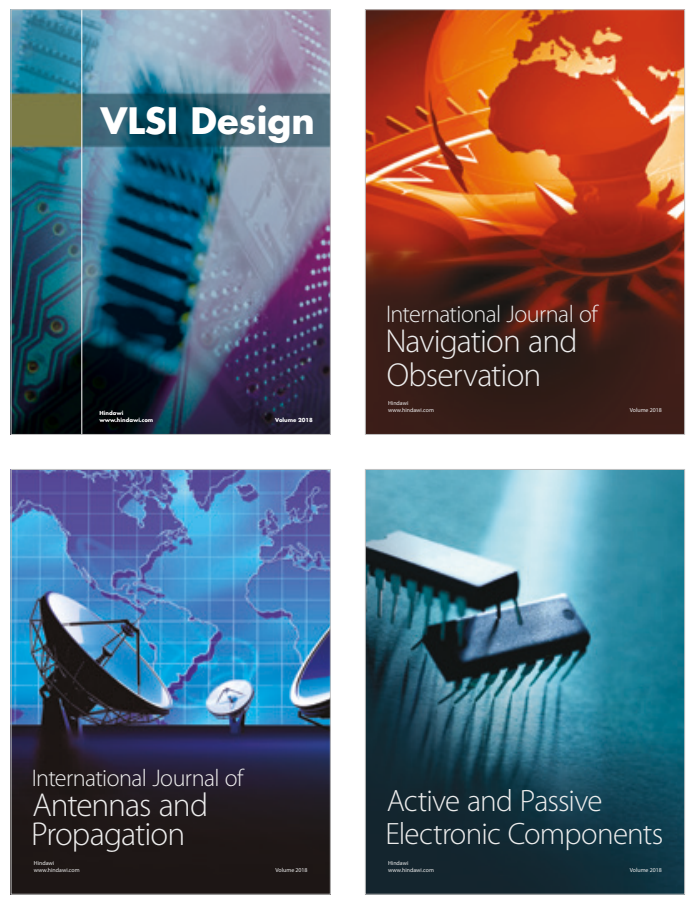
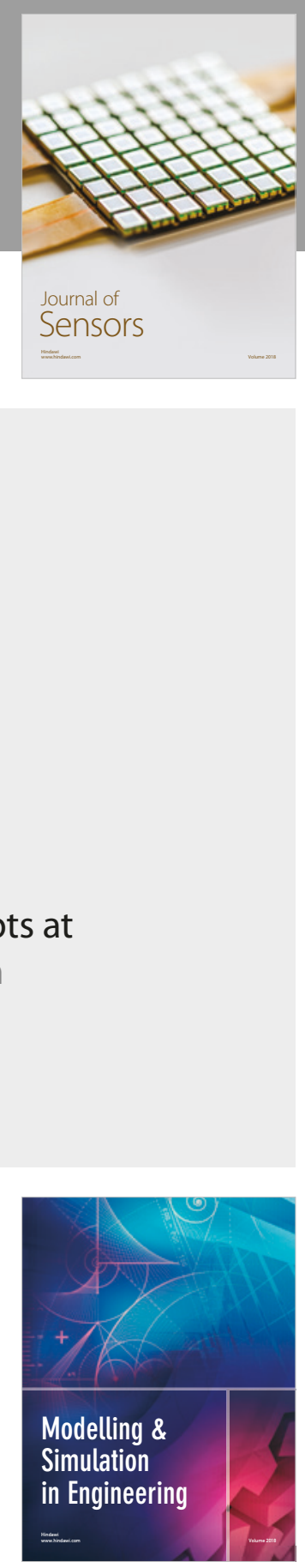

\section{Advances \\ Multimedia}
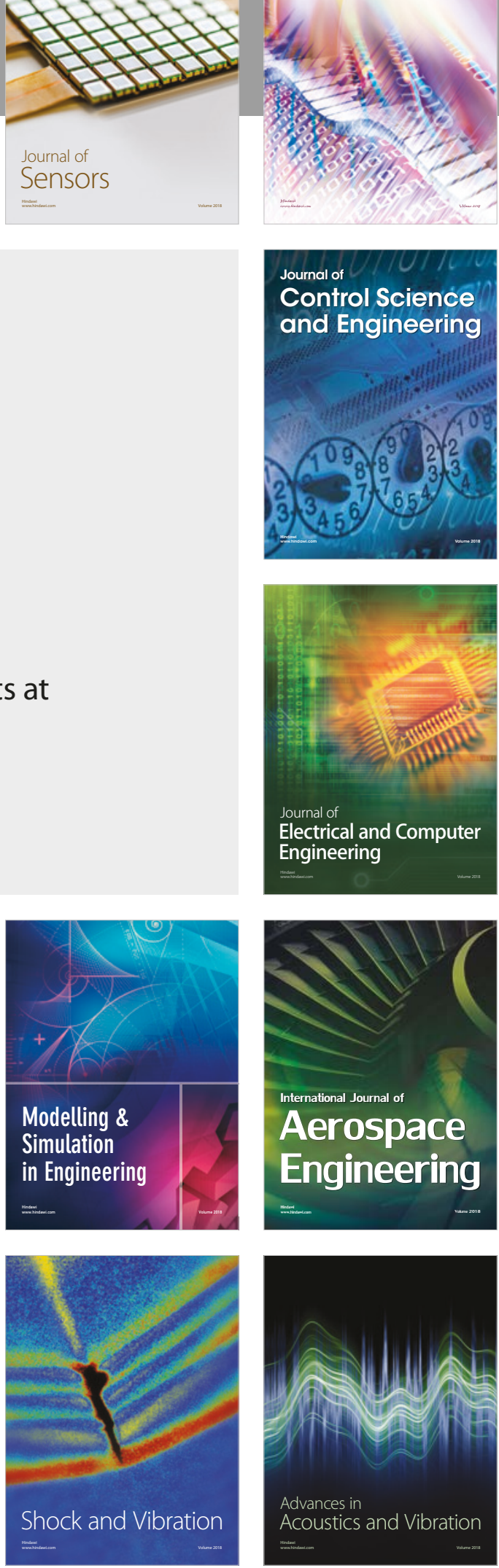